ARTí́CUlO

\title{
Interacciones tróficas de Australoheros facetus (Perciformes: Cichlidae), pez exótico en el humedal El Culebrón, Coquimbo, Chile
}

Trophic interactions of Australoheros facetus (Perciformes: Cichlidae), exotic fish in the El Culebrón wetland, Coquimbo, Chile

\section{Jorge Avilés ${ }^{*}$, Amanda López ${ }^{2}$ y Héctor Flores ${ }^{3}$}

\author{
${ }^{1}$ Sala de Colecciones Biológicas, Facultad de Ciencias del Mar, Universidad Católica del Norte, Larrondo 1281, Coquimbo, \\ Chile \\ ${ }^{2}$ Colegio Guillermo Cereceda Rojas, Departamento de Educación Municipal Coquimbo, Santiago Amengual 364, San Juan, \\ Coquimbo, Chile \\ ${ }^{3}$ Departamento de Acuicultura, Facultad de Ciencias del Mar, Universidad Católica del Norte, Larrondo 1281, Coquimbo, \\ Chile \\ *Autor corresponsal: javiles2006@hotmail.com
}

\begin{abstract}
Australoheros facetus is distributed in coastal streams of Uruguay and Rio Grande do Sul in Brazil, Basin of the Paraná River and lower region of the Paraná River in Argentina, with a great capacity of adaptation to the environment. It is of interest to the Aquarium and product of this activity has produced releases in South America and Europe. In Chile, its introduction dates back to 1940. This species was detected living in the El Culebrón wetland, Coquimbo, Chile and questions arise as to what will be the impact of this introduced species on native species, especially if the river shrimp Cryphiops caementarius is preyed. To obtain information about their feeding habits, and using citizen science with a group of students from the Guillermo Cereceda Rojas school in Coquimbo, 3 samplings were conducted between 2015 and 2016; 24 individuals were captured with $37.5 \%$ of females, the smallest specimens and the heaviest were males. The fish caught were recorded their total length $\left(L_{t}\right)$, total weight $\left(P_{t}\right)$ and sex. The proportion of sex, size distribution, $L_{t} / P_{t}$ ratio was determined. Their stomach content was analyzed, applying the frequency of occurrence, numerical and gravimetric indexes, the relative importance index (RII) and its respective percentage were determined. An isometric growth was determined and there were no differences in the $L_{t} / P_{t}$ ratio for females and males. The presence of 20 items was recorded (remains of plants, Annelidae, Mollusca, Arthropoda and Pisces). The most important prey were fish (37.5\% RII), Cryphiops caementarius (25\% RII) and Chironomidaes ( $13.8 \%$ RII). It is recommended to evaluate the presence of this species, in other hydrographic systems of the region and especially, the impact of $A$. facetus on the predation of river shrimp.
\end{abstract}

Key words: Fish, feeding, prey, conservation, introduction of species, citizen science

Resumen.- Australoheros facetus se distribuye en los arroyos costeros de Uruguay y Río Grande do Sul en Brasil, Cuenca del río Paraná y región inferior del río Paraná en Argentina, con una gran capacidad de adaptación al ambiente. Esta especie es de interés para la acuariofilia y producto de esta actividad se han producido liberaciones en Sudamérica y Europa. En Chile, su introducción data desde 1940. Esta especie fue detectada habitando en el humedal El Culebrón, Coquimbo, Chile y surgen las preguntas de cuál será el impacto de esta especie introducida en las especies nativas, especialmente si su presa es el camarón de río Cryphiops caementarius. Para obtener información de sus hábitos alimentarios, junto con un grupo de estudiantes del colegio Guillermo Cereceda Rojas de Coquimbo, se efectuaron 3 muestreos entre el 2015 y 2016. Se capturaron 24 individuos con un 37,5\% de hembras, los ejemplares más pequeños y los de mayor peso fueron machos. A los peces capturados se les registró su longitud total $\left(\mathrm{L}_{t}\right)$, peso total $\left(\mathrm{P}_{\mathrm{t}}\right)$ y sexo. Se determinó la proporción de sexo, distribución de talla, relación $L_{t} / P_{t}$. Se analizó su contenido estomacal, aplicando los índices de frecuencia de ocurrencia, numérica y gravimétrica, determinando el índice de importancia relativa (IIR) y su respectivo porcentaje. Se obtuvo como resultado un crecimiento isométrico y no hubo diferencias en la relación $L_{t} / P_{t}$ para hembras y machos. Se registró la presencia de 20 ítem presa (restos de plantas, Annelidae, Mollusca, Arthropoda y Pisces). Las presas mas importantes fueron peces $(37,5 \%$ IIR), Cryphiops caementarius ( $25 \%$ IIR) y Chironomidaes ( $13,8 \%$ IIR). Se recomienda evaluar la presencia de esta especie, en otros sistemas hidrográficos de la región y en especial, el impacto de $A$. facetus en la depredación de camarón de río.

Palabras clave: Peces, alimentación, presa, conservación, introducción, ciencia ciudadana 


\section{INTRODUCCIÓN}

Australoheros facetus (Jenyns, 1842), conocido como chanchito, palometa negra, o castañeta, se distribuye en los arroyos costeros de Uruguay y Río Grande do Sul en Brasil, Cuenca del río Paraná y región inferior del río Paraná en Argentina (Froese \& Pauly 2017). Los ejemplares con distribución más austral reportados corresponden a los $38^{\circ} \mathrm{S}$ en Argentina (Casciotta et al. 1999).

Es una especie eurotópica (Gómez 1996), con gran capacidad de maniobra que le permite adaptarse muy bien en ambientes estructuralmente complejo (Gómez et al. 2003). Es omnívora y tiene cuidado parental (Ruiz \& Marchant 2004) ${ }^{1}$. Es una especie de interés para la acuariofilia y producto de esta actividad es que se han producido liberaciones en diferentes lugares y países, no solo de Sudamérica donde esta especie es nativa, sino que también en Europa (Ribeiro et al. 2007).

Su introducción en Chile, fue registrada en 1940 en el lago Peñuelas, Valparaíso, Chile (Iriarte et al. 2005), posiblemente desde Argentina (De Buen 1959); sin embargo, se desconoce como se efectuó específicamente la introducción de esta especie en Chile (Arratia 1978). También hay registros en las lagunas aledañas a Concepción (Laguna Grande, y Chica de San Pedro, La Posada, Las Tres Pascualas, entre otras), cuya presencia se debe a la liberación efectuada por personas que mantenían algunos ejemplares en acuario (Ruiz \& Marchant 1989); para el norte del país se encuentra reportado hasta Pichidangui, Región de Coquimbo (Iriarte et al. 2005).

En el 2009 se detectó la presencia de A. facetus en el Humedal El Culebrón (Rivera et al. 2009)². Este humedal se ubica en el extremo sur-oeste de la bahía de Coquimbo, la cuenca es del tipo preandina exorreica con régimen pluvial, su caudal promedio es de $0,38 \mathrm{~m}^{3} \mathrm{~s}^{-1}$, con aportes naturales desde una serie de quebradas y de manera artificial recibe los excedentes de riego del Canal Bellavista, este último conduce aguas desde el Río Elqui hacia el sector de Pan de Azúcar; la zona baja del estero recibe aportes de instrucciones salinas, tanto para los depósitos de playa, como para el acuífero fluvial en las inmediaciones de la desembocadura (Rivera et al. 2009). El hallazgo de Australoheros facetus motiva preguntas, ¿Cómo llegaron estos ejemplares?, ¿Cuál será su interacción trófica con el camarón de río, Cryphiops caementarius (Molina, 1782)? Esta última es una especie nativa, extraída por camaroneros y en categoría vulnerable (MMA 2017); se desconoce el impacto de la introducción de esta especie en este humedal, que es un refugio para la vida silvestre en la Provincia de Elqui. Para dilucidar estas interrogantes, el objetivo de este trabajo fue describir la dieta y hábitos alimentarios de $A$. facetus, con el fin de recopilar antecedentes para establecer bases de la ecología trófica de esta especie introducida.

\section{Materiales y MÉtodos}

Se efectuaron 3 campañas de muestreo en terreno, en mayo y octubre 2015 y marzo 2016, en el humedal El Culebrón, Coquimbo (29 $57^{\prime} \mathrm{S}, 7^{\circ} 19^{\prime} \mathrm{W}$ ) (Fig. 1), con participación de los estudiantes del Colegio Guillermo Cereceda Rojas de Coquimbo.

Para la captura de los peces se empleó un chinguillo (mango: $1 \mathrm{~m}$, sacadera: $40 x 40 \mathrm{~cm}$, luz de malla: $1 \mathrm{~mm}$ ) y una red de arrastre manual (paño de $5 \times 1,5 \mathrm{~m}$; luz de malla 5 $\mathrm{mm}$ ). Se efectuaron lances al azar a una profundidad de entre 0,5 y $1,5 \mathrm{~m}$ de profundidad.

Los peces capturados (24 ejemplares) fueron trasladados a la Sala de Colecciones de la Facultad de Ciencias del Mar, donde se conservaron en formalina al $10 \%$. Posteriormente, a cada ejemplar se registró su peso total $\left(\mathrm{P}_{\mathrm{t}}\right)$ y eviscerado $\left(\mathrm{P}_{\mathrm{e}}\right)$, con una balanza semianalítica marca AND modelo GX 1500 precisión 0,1 g y se midió su longitud total $\left(\mathrm{L}_{\mathrm{t}}\right)$ con un ictiómetro de precisión $0,01 \mathrm{~cm}$. Los peces fueron disectados, determinando el sexo y el tubo digestivo fue guardado en bolsa plástica con alcohol al 70\% para su posterior análisis.

Se evaluó la proporción de sexos mediante una prueba de Chi-cuadrado para la relación esperada de 1:1 entre hembras y machos (Zar 1999). Se comparó para machos y hembras las curvas potenciales de la relación $\mathrm{L}_{\mathrm{t}} / \mathrm{P}_{\mathrm{t}}$, mediante un análisis de covarianza (Zar 1999). Se evaluó la significancia estadística del exponente isométrico (b), éste se analizó con la función propuesta por Pauly (1984):

$$
t=\frac{\text { s.d. }(x)}{\text { s.d. }(y)} * \frac{\left|b_{i}-3\right|}{\sqrt[2]{1-r^{2}}} * \sqrt[2]{n_{i}-2}
$$

donde, $t$ es el estadístico t-student; s.d.(x) e $(y)$ corresponden a la desviación estándar del logaritmo de la $\mathrm{L}_{\mathrm{t}}$ y del $\mathrm{P}_{\mathrm{t}}$ para cada grupo analizado; $\mathrm{n}_{\mathrm{i}}$ es el número de peces muestreados; $b_{i}$ es el valor ajustado de $b$ y $r^{2}$ es el ajuste potencial del coeficiente de determinación. 
Se determinó el factor de condición de Fulton por la relación $\mathrm{K}=\mathrm{P}_{\mathrm{t}}^{*} 100 / \mathrm{L}_{\mathrm{t}}^{3}$ (Nikolsky 1963). Para comparar este índice entre hembras y machos, se aplicó una ANOVA, previa evaluación de la normalidad y homocedasticidad de los datos.

Para analizar la dieta, el contenido estomacal de los 24 ejemplares capturados, se dispuso en cápsulas de Petri, procediendo a separar los diferentes ítems, a contar las distintas presas y a identificarlas mediante claves especializadas, hasta el nivel más específico posible. Separadas las presas, se efectuaron pesajes agrupando a todos los individuos de un mismo ítem, mediante una balanza analítica marca AND modelo GR-202 precisión $0,0001 \mathrm{~g}$.
Para el análisis de la dieta, se determinó los porcentajes de frecuencia numérica $(\% \mathrm{~N})$, de ocurrencia (\%FO) y gravimétrica (\%P) (Hyslop 1980).

Para representar la importancia de las presas en la alimentación de los peces analizados y a manera de resumir los métodos anteriores, se calculó el índice de importancia relativa (IIR) (Pinkas et al. 1971), por la relación IIR= $\% \mathrm{~N}$ $+\% \mathrm{P}) * \% \mathrm{FO}$. Paralelamente, y para obtener una información general y balanceada de la categoría de la presa, se aplicó el \%IIR (Cortes 1997).

Los ejemplares de $A$. facetus quedaron incorporados en la Sala de Colecciones Biológicas de la Facultad de Ciencias del Mar de la Universidad Católica del Norte, bajo el registro SCBUCN 7329.

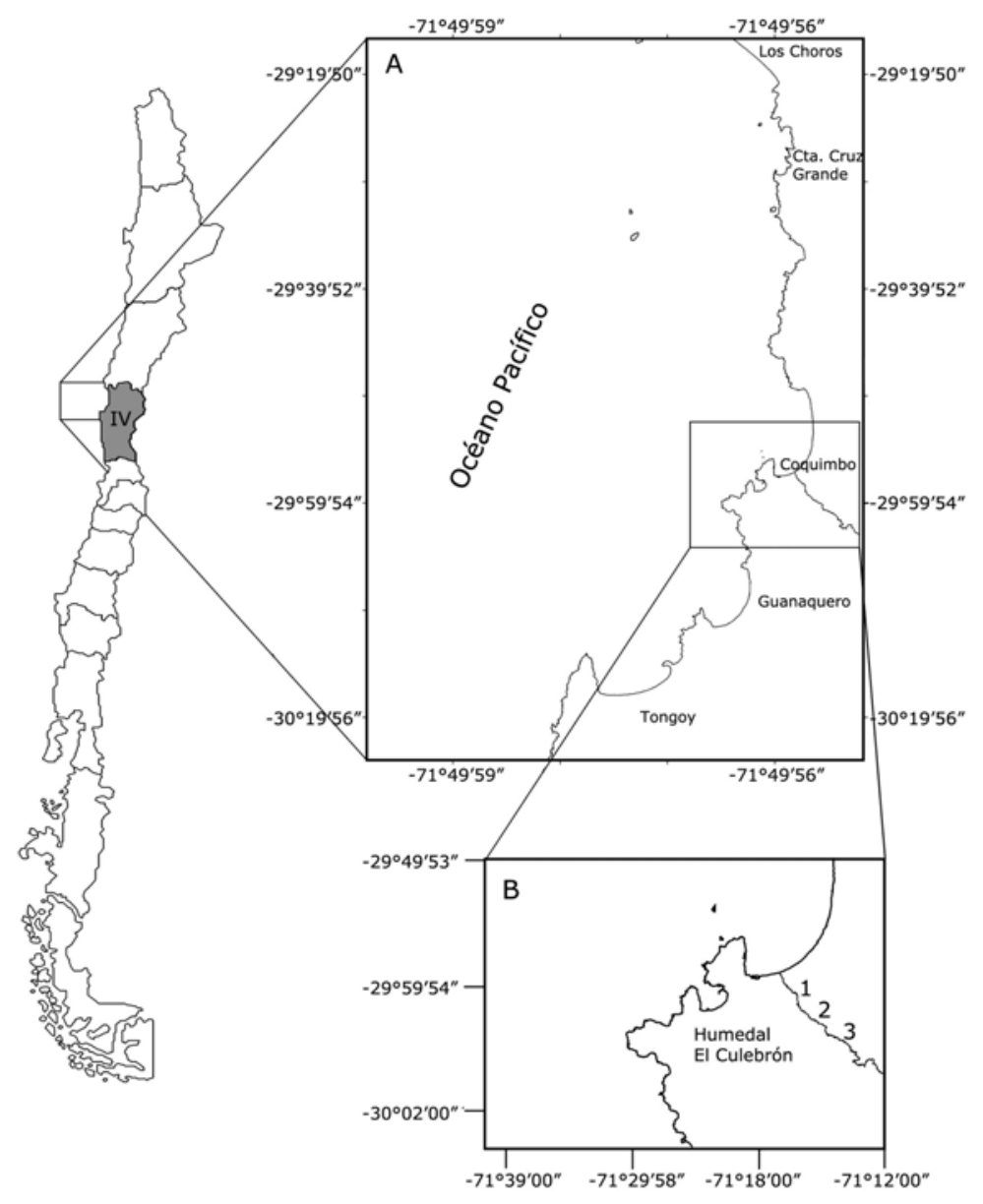

Figura 1. Humedal El Culebrón, Coquimbo, Chile. A) Localización geográfica; B) distribución espacial de las estaciones de muestreo (1, 2 y 3) / Study area, El Culebrón wetland, Coquimbo, Chile. A) Geographical location; B) spatial distribution of sampling stations (1, 2 and 3) 


\section{Resultados}

Del total de 24 ejemplares, cuya $\mathrm{L}_{\mathrm{t}}$ fluctuó entre 5,2 y 13,5 cm y el $P_{t}$ entre 2,7 y 47,4 g (Tabla 1), la proporción hembra $(37,5 \%)$ y machos $(62,5 \%)$ fue de $1: 1(P<0,05$; Tabla 2$)$. En las campañas de muestreos fue igual proporción para octubre 2015 y marzo $2016(P<0,005)$, mientras que en mayo 2015 se capturó sólo machos. Los ejemplares más pequeños $\left(2,3 \mathrm{~cm} \mathrm{~L}_{\mathrm{t}}\right)$ y los de mayor peso $(47,4 \mathrm{~g} \mathrm{P}$ ) corresponden a machos (Fig. 2).

La relación $\mathrm{L}_{\mathrm{t}} / \mathrm{P}_{\mathrm{t}}$ se ajustó a un modelo potencial, definido por la relación: $\mathrm{P}_{\mathrm{t}}=0,0164 \mathrm{~L}_{\mathrm{t}}^{3,049}$ (Fig. 3), no existiendo diferencias estadísticas entre sexos (ANCOVA, pendiente: $\mathrm{Ft}=2,09 ; \mathrm{Fc}=0,42$; intercepto: $\mathrm{Ft}=2,08 ; \mathrm{Fc}=$ $0,49)$. La pendiente de la función potencial para ambos sexos $(b=3,049)$, fue estadísticamente igual a $3(\mathrm{t}=0,627$; $P<0,05)$, lo que determinó un crecimiento isométrico, igual relación se mantiene entre ambos sexos (hembras $\mathrm{t}=$ 0,808; machos $\mathrm{t}=0,243 ; P £ 0,05)$. El índice de Condición de Fulton entre hembras y machos, tuvo distribución normal, sin embargo no homocedasticidad, por tanto un

Tabla 1. Longitud total (Lt), peso total (Pt), desviación estándar (D.E.), valores mínimos y máximos de Australoheros facetus capturados en el humedal El Culebrón, Coquimbo, en mayo y octubre 2015 y marzo 2016 / Total length (Lt), total weight (Pt), standard deviation (SD), minimum and maximum values of Australoheros facetus captured in the El Culebrón wetland, Coquimbo, in May and October 2015 and March 2016

\begin{tabular}{lcccc}
\hline & Promedio & D.E. & Mínimo & Máximo \\
\hline $\mathrm{Lt}(\mathrm{cm})$ & 8,3 & 2,4 & 5,2 & 13,5 \\
$\mathrm{Pt}(\mathrm{g})$ & 13,5 & 2,4 & 2,7 & 47,4 \\
\hline
\end{tabular}

Tabla 2. Proporción sexual (\%) en cada muestreos de Australoheros facetus capturados en el humedal El Culebrón, Coquimbo, en mayo y octubre 2015 y marzo 2016. / Sexual proportion (\%) in each sample of Australoheros facetus captured in the El Culebrón wetland, Coquimbo, in May and October of 2015 and March of 2016. N: number of captured specimens

\begin{tabular}{lccc}
\hline & $\mathrm{N}$ & $\begin{array}{c}\text { Hembras } \\
(\%)\end{array}$ & $\begin{array}{c}\text { Machos } \\
(\%)\end{array}$ \\
\hline Mayo 2015 & 4 & 0,0 & 100,0 \\
Octubre 2015 & 5 & 60,0 & 40,0 \\
Marzo 2016 & 15 & 40,0 & 60,0 \\
Total & 24 & 37,5 & 62,5 \\
\hline
\end{tabular}

$\mathrm{N}$ : número de ejemplares capturados
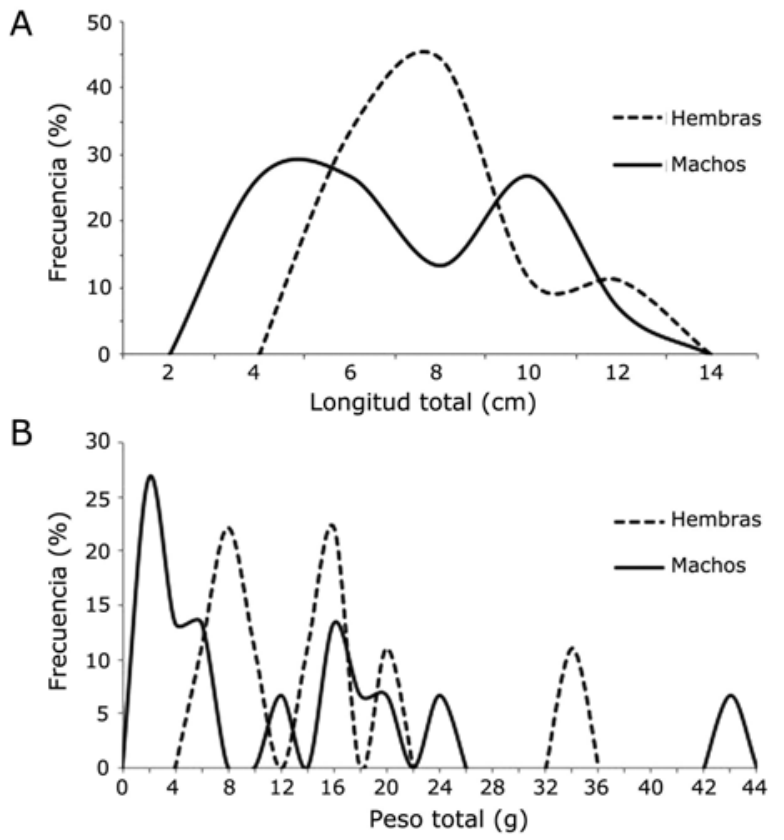

Figura 2. Distribución en longitud total (A) y peso total (B) en hembras y machos de Australoheros facetus capturados en el Humedal El Culebrón en mayo y octubre 2015 y marzo 2016 / Distribution in total length $(A)$ and total weight $(B)$ in females and males of Australoheros facetus captured in El Culebrón Wetland in May and October 2015, and March 2016

ANOVA no paramétrico, permitió inferir que no hubo diferencias estadísticamente significativas entre hembras $(0,016 \pm 0,001)$ y machos $(0,017 \pm 0,001)$.

El 100\% de los peces contenía presas y restos de alimento. Se registró la presencia de 20 ítem presa, que incluyen restos de plantas, Annelidae, Mollusca, Arthropoda y Pisces. El grupo taxonómico que aportó en riqueza más al consumo de $A$. facetus fue Arthropoda con 11 ítems, seguido de 4 ítems para Peces.

Las presas más frecuentes (FO), corresponden a Chironomidae (66,7\%), Cryphiops caementarius, Tipulidae (50,0\%), restos de peces (45,8\%), Acari (41,7\%) y Gambusia holbrooki (alevines) (29,2\%), respectivamente. El resto de las presas presentó valores inferiores al 29,0\% (Tabla 3).

En número de presas, Chironomidae fue el ítem más importante (12,9\%), seguido por C. caementarius (12,2\%), Tipulidae (9,5\%), Acari (8,2\%), G. holbrooki (alevines, $8,2 \%)$ y restos de peces $(8,2 \%)$. El resto de las presas presentó valores inferiores al 6\% (Tabla 3). 
Figura 3. Relación longitud total $\left(L_{t}\right)$ y peso total $\left(P_{t}\right)$ en Australoheros facetus para el humedal El Culebrón en mayo y octubre 2015 y marzo 2016 / Total length ratio $\left(L_{t}\right)$ and total weight $\left(\mathrm{P}_{t}\right)$ in Australoheros facetus for the El Culebrón wetland in May and October 2015, and March 2016

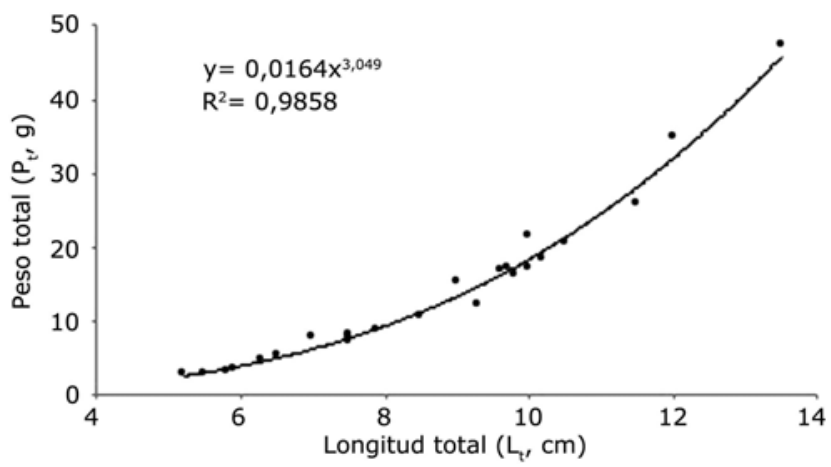

Las presas más consumidas en peso corresponde a restos de peces (26,6\%), Cyprinus carpio (alevines) (21,7\%), C. caementarius (20,3\%), G. holbrooki (alevines) $(14,7 \%)$ y Mugil cephalus (alevines) (7,9\%). El espectro trófico restante tuvo incidencias que no llegaron al $2 \%$ (Tabla 3).

En base al IIR, las presas más importantes en la dieta de A. facetus fueron peces, C. caementarius y Chironomidaes (Tabla 3). Al agrupar todos los peces consumidos por $A$. facetus, éstos representan un $37,5 \%$ del IIR\%, seguido de C. caementarius con un $25 \%$ y Chironomidaes con un $13,8 \%$.

Tabla 3. Frecuencia numérica (\%N), de ocurrencia (\%FO), gravimétrica (\%P) e índice de importancia relativa (IIR) de Australoheros facetus en el humedal El Culebrón, en mayo y octubre 2015 y marzo 2016. N: número de individuos/ítem; P: peso/item / Numerical frequency (\% N), occurrence (\% FO), gravimetric (\% P) and relative importance index (IIR) of Australoheros facetus in the El Culebrón wetland, in May and October 2015, and March 2016. N: number of individuals/item; P: weight/item

\begin{tabular}{|c|c|c|c|c|c|c|c|c|}
\hline Especie / Items & $\mathrm{N}$ & $\% \mathrm{~N}$ & FO & $\% \mathrm{FO}$ & $\mathrm{P}(\mathrm{g})$ & $\% \mathrm{P}$ & IIR & $\%$ IIR \\
\hline \multicolumn{9}{|l|}{ Plantae } \\
\hline Restos algas filamentosas & 6 & 4,08 & 4 & 16,16 & 0,0002 & 0,13 & 0,68 & 1,05 \\
\hline Restos vegetales & 8 & 5,44 & 6 & 25,00 & 0,0004 & 0,25 & 1,42 & 2,19 \\
\hline \multicolumn{9}{|l|}{ Annelidae } \\
\hline Hirudinea & 3 & 2,04 & 2 & 8,33 & 0,0007 & 0,44 & 0,21 & 0,32 \\
\hline \multicolumn{9}{|l|}{ Mollusca } \\
\hline Lymnaeidae & 5 & 3,40 & 3 & 12,5 & 0,0022 & 1,39 & 0,60 & 0,92 \\
\hline Physa chilensis & 4 & 2,72 & 3 & 12,5 & 0,0012 & 0,76 & 0,43 & 0,67 \\
\hline \multicolumn{9}{|l|}{ Arthropoda } \\
\hline Acari & 12 & 8,16 & 10 & 41,66 & 0,0009 & 0,57 & 3,64 & 5,60 \\
\hline Hyalela sp. & 5 & 3,40 & 4 & 16,66 & 0,0018 & 1,13 & 0,76 & 1,16 \\
\hline Cryphiops caementarius & 18 & 12,24 & 12 & 50,00 & 0,0322 & 20,28 & 16,26 & 25,02 \\
\hline Poduridae & 4 & 2,72 & 4 & 16,66 & 0,0008 & 0,50 & 0,54 & 0,83 \\
\hline Chironomidae & 19 & 12,93 & 16 & 66,66 & 0,0008 & 0,50 & 8,95 & 13,78 \\
\hline Tipulidae & 14 & 9,52 & 12 & 50,00 & 0,0009 & 0,57 & 5,05 & 7,76 \\
\hline Culex sp. & 8 & 5,44 & 4 & 16,66 & 0,0007 & 0,44 & 0,98 & 1,51 \\
\hline Belostomatidae & 2 & 1,36 & 2 & 8,33 & 0,0004 & 0,25 & 0,13 & 0,21 \\
\hline Elmidae & 3 & 2,04 & 2 & 8,33 & 0,0012 & 0,76 & 0,23 & 0,36 \\
\hline Liodessus sp. & 3 & 2,04 & 1 & 4,16 & 0,0011 & 0,69 & 0,11 & 0,17 \\
\hline Thermonectus sp. & 5 & 3,40 & 4 & 16,66 & 0,0008 & 0,50 & 0,65 & 1,00 \\
\hline \multicolumn{9}{|l|}{ Chordata } \\
\hline $\begin{array}{l}\text { Gambussia holbrooki } \\
\text { (alevines) }\end{array}$ & 12 & 8,16 & 7 & 29,16 & 0,0233 & 14,67 & 6,66 & 10,25 \\
\hline Mugil cephalus (alevines) & 3 & 2,04 & 2 & 8,33 & 0,0126 & 7,93 & 0,83 & 1,28 \\
\hline Cyprimus carpio (alevines) & 1 & 0,68 & 1 & 4,16 & 0,0344 & 21,66 & 0,93 & 1,43 \\
\hline $\begin{array}{l}\text { Restos peces } \\
\text { (indeterminados) }\end{array}$ & 12 & 8,16 & 11 & 45,83 & 0,0422 & 26,57 & 15,92 & 24,50 \\
\hline
\end{tabular}




\section{Discusión}

Australoheros facetus tiene un importante valor como pez ornamental (Castello 1972); es el cíclido más austral del mundo, tolerando aguas templadas-frías $\left(>4^{\circ} \mathrm{C}\right)$, y salinas (>20 g L-1 ; Gómez 1996, Gómez \& González 2006). Es una especie que se adapta con facilidad al ambiente, tiene una capacidad reproductiva de mucho éxito, como es el cuidado parental (Ruiz \& Marchant 2004).

Al comparar los índices biológicos entre los ejemplares capturados en el humedal El Culebrón, con otros cuerpos de agua, se constató que el coeficiente de alometría (b) fue semejante a lo reportado para esta especie en Argentina, Brasil y Uruguay, con un intervalo de confianza que va entre 2,54 y 3,52 (Teixeirade Mello et al. 2009, Antonetti et al. 2014, Gómez 2014, Mendonça et al. 2015). El Índice de Condición de Fulton reportado para el humedal El Culebrón $(1,834 \pm 0,175)$ fue inferior al reportado por Gómez (2014) para Formosa, Argentina (3,903 \pm 0,6139); esta diferencia se debería al tamaño de los peces de ambas muestras. Los peces argentinos fueron de un peso promedio de 2,46 g, mientras que los capturados en el humedal El Culebrón tuvieron un peso promedio de 13,45 g.

Australoheros facetus es un pez omnívoro, que en Uruguay (Lago Rodó, Montevideo) se alimenta principalmente de larvas y pupas de Chironomidae, peces (Cnesterodon decemmaculatus Jenyns, 1842) y vegetales (algas, periphyton y macrofitas), mientras que los individuos de mayor talla incorporan en la dieta a Palaemonetes argentinus (Yafé et al. 2002). En Argentina (Río Primero, Cordova) consume principalmente Copepoda, larvas de Chironomidae y algas (Gutiérrez et al. 1986). En Chile (Laguna Grande y Chica, Concepción) hay registro de consumo de Chironomidae (37,5\%), Peces (Galaxias maculatus, Cheirodon galusdae, G. holbrooki, 18,7\%), Ostracoda (10,5\%) y un 4,3\% de Samastacus (Ruiz \& Marchant 1988, 2004). Los resultados sugieren que $A$. facetus es una especie con un amplio espectro trófico, donde Ostracodos, Copepoda y Chironomidae son ítems importantes en tallas pequeñas, y a medida que van creciendo incorporan peces y crustáceos mayores como Palaemonetes argentinus (Uruguay), y en Chile Samastacus sp. (sur) y Cryphiops caementarius (centronorte).

En el caso de su introducción en Chile, A. facetus es una especie que compite con especies nativas (Iriarte et al. 2005), ocasionando impactos tanto en peces como invertebrados (Ruiz \& Marchant 2004). Su permanencia en el humedal El Culebrón, es favorecida por su tolerancia a salinidad y resistencia a temperaturas bajas (Gómez 1996). El acuífero que proporciona agua al humedal se caracteriza por una salinidad moderada de $10,7 \mathrm{mg} \mathrm{L}^{-1}$ (Cárdenas-Castillero 2017); estas condiciones demuestran el grado exitoso de adaptación al ambiente de esta especie, que tolera salinidades superiores a 20 , característica que sería un relicto fisiológico de sus ancestros marinos (Gómez \& González 2006). Esta especie no ha sido registrada en otros humedales en las regiones de Valparaíso y Coquimbo (Figueroa et al. 2009). Su presencia en el humedal El Culebrón, se debería a una situación semejante a la ocurrida en Portugal, donde hubo dispersión y translocación por personas (Ribeiro et al. 2009), mientras que en España su introducción fue local y estable (Elvira 1997, Durán et al. 2014).

Las especies invasoras, se consideran una amenaza, alterando la biodiversidad, fluctuaciones en los ecosistemas e impactos económicos en la agricultura, pesca y comercio (Lovell et al. 2006, Aguirre \& Mendoza 2009). Australoheros facetus es una especie exótica, que se ha adaptado muy bien a las condiciones hídricas de Chile central, con una estrategia reproductiva de cuidado parental, que junto a su adaptación al ambiente, podría explicar el éxito selectivo de colonización de nuevos cursos de agua. Bajo estas consideraciones, es necesario futuros estudios en otros sistemas hidrográficos de la región, que permitan evaluar la presencia de esta especie y en especial, el impacto de A. facetus en la depredación de camarón de río Cryphiops caementarius, donde este recurso constituye un importante sustento para pescadores artesanales.

\section{Agradecimientos}

Se agradece a los estudiantes Lidia Veliz Martínez, Gian Jeraldo Alvarado, Millaray Pizarro Álvarez, Constanza Pizarro Laguez y Cristopher Cortés Rojas del Colegio Guillermo Cereceda Rojas de Coquimbo, por su interés y participación en las campañas de muestreo. Trabajo dedicado a la memoria del Dr. Roberto Meléndez.

\section{LITERATURA CITADA}

Aguirre MA \& R Mendoza. 2009. Especies exóticas invasoras: impactos sobre las poblaciones de flora y fauna, los procesos ecológicos y la economía. En: J Sarukhan, R Dirzo, R González \& I March (eds). Capital natural de México, Vol. 2: Estado de conservación y tendencias de cambio, pp. 277-318. Comisión Nacional para el Conocimiento y Uso de la Biodiversidad, México. 
Antonetti DA, ME Leal \& UH Schulz. 2014. Lengthweight relationships for 19 fish species from the Jacuí Delta, RS, Brazil. Journal of Applied Ichthyology 30(1): 259-260.

Arratia G. 1978. Comentario sobre la introducción de peces exóticos en aguas continentales de Chile. Ciencias Forestales 1: $21-30$.

Cárdenas-Castillero G. 2017. Evaluación de la salinidad de la subcuenca Oeste del acuífero El Culebrón. Tesis de Maestría, Universidad La Sorbonne, Universidad Pierre et Marie Curie (París VI), París, 209 pp.

Casciotta JR, AAlmirón, A Cione \& MM Azpelicueta. 1999. Brazilian freshwater fish assemblages from southern Pampean area, Argentina. Biogeographica 75: 67-78.

Cortes E. 1997. A critical review of methods of studying fish feeding based on analysis of stomach contents: application to elasmobranch fishes. Canadian Journal of Fisheries and Aquatic Sciences 54: 726-738.

De Buen F. 1959. Los peces exóticos en las aguas dulces de Chile. Investigaciones Zoológicas Chilenas 5: 103-138.

Durán C, M Lanao \& AAnadón. 2014. Actuaciones contra las invasiones acuáticas en la Confederación Hidrográfica del Ebro. Ambienta 109: 42-51.

Elvira B. 1997. Impacto y control de los peces exóticos introducidos en España. En: Granado-Lorencio C (ed). Conservación, recuperación y gestión de la ictiofauna continental ibérica. Publicaciones de la Estación de Ecología Acuática, Sevilla: 139-151.

Froese R \& D Pauly. 2017. FishBase. <www.fishbase.org>

Gómez SE. 1996. Resistenza alla temperatura e salinit in pesci della provincia di Buenos Aires (Argentina), con implicazioni zoogeografiche. En: Atti 4 Convegno Nazionale Associacione Italiana Ittiologica Acque Dolci, Trento, pp. 171-172.

Gómez SE. 2014. Las relaciones longitud peso en algunos peces tropicales de acuario. Revista AquaTIC 41: 1-7.

Gómez SE \& MJ González. 2006. Resistencia a la salinidad en dos especies de peces neotropicales de la familia Cichlidae (Pisces, Perciformes). Memoria de la Fundación La Salle de Ciencias Naturales 166: 47-57.

Gómez SE, J González \& A Giusto. 2003. Velocidad de natación de un especialista en maniobra, Cichlasoma facetum (Jenyns) (Pisces, Cichlidae), en condiciones experimentales. Revista Museo Argentino Ciencias Naturales 5(1): 87-92.

Hyslop EJ. 1980. Stomach contens analysis. A review of methods and their applications. Journal of Fish Biology 17: 411-429.
Iriarte JA, GA Lobos \& FM Jaksic. 2005. Invasive vertebrate species in Chile and their control and monitoring by governmental agencies. Revista Chilena de Historia Natural 78: 143-154.

Lovell S, S Stone \& L Fernandez. 2006. The economic impacts of aquatic invasive species: A review of the literature. Agricultural and Resource Economics Review 35(1): 195-208.

Mendonça HS, D SilvaCamacho, SM Pinto \& FG Araújo. 2015. Lengthweight relationships of 14 fish species from a lowland tropical reservoir in southeastern Brazil. Journal of Applied Ichthyology 31(5): 970-972.

MMA. 2017. Cryphiops caementarius (Molina, 1782). Sistema de Inventario de Especies Silvestres. Ministerio Medio Ambiente, Gobierno de Chile, Santiago. <http:// especies.mma.gob.cl>

Nikolsky GV. 1963. The ecology of fishes, 352 pp. Academic Press, New York.

Pauly D. 1984. Fish population dynamics in tropical waters: a manual for the use with programmable calculators. ICLARM Studies and Reviews 8: 1-325.

Pinkas L, MS Oliphant \& ILK Iverson. 1971. Food habits of albacore, Bluefin tuna, and bonito in California waters. Fish and Game Bulletin 152: 1-105.

Ribeiro F, RL Orjuela, MF Magalhães \& MJ CollaresPereira. 2007. Variability in feeding ecology of a South American cichlid: a reason for successful invasion in mediterranean-type rivers? Ecology of Freshwater Fish 16: 559-569.

Ribeiro F, MJ CollaresPereira \& PB Moyle. 2009. Nonnative fish in the fresh waters of Portugal, Azores and Madeira Islands: a growing threat to aquatic biodiversity. Fisheries Management and Ecology 16(4): 255-264.

Ruiz V \& M Marchant. 1989. Sobre Cichlasoma facetum (Jenyns, 1842) (Perciformes, Cichlidae) de las lagunas Grande y Chica de San Pedro, VIII Región, Chile. Boletín de la Sociedad de Biología de Concepción, Chile 60: 227229.

Teixeirade Mello F, N Vidal, G Eguren \& M Loureiro. 2009. Length-weight relationships of 21 fish species from the lower section of the Santa Lucia river basin (CanelonesMontevideo, Uruguay). Journal of Applied Ichthyology 25(4): 491-492.

Recibido el 27 de marzo de 2018 y aceptado el 25 de julio de 2018

Editor: Claudia Bustos D. 